\title{
Beneficial effects of the CorCap cardiac support device: Five-year results from the Acorn Trial
}

\author{
Douglas L. Mann, MD, ${ }^{\mathrm{a}}$ Spencer H. Kubo, MD, ${ }^{\mathrm{b}}$ Hani N. Sabbah, PhD, ${ }^{\mathrm{c}}$ Randall C. Starling, MD, ${ }^{\mathrm{d}}$ \\ Mariell Jessup, MD, ${ }^{\mathrm{e}}$ Jae K. Oh, MD, ${ }^{\mathrm{f}}$ and Michael A. Acker, MD
}

Background: The CorCap cardiac support device (Acorn Cardiovascular, Inc, St Paul, Minn) is the first device that specifically addresses ventricular remodeling in heart failure by reducing wall stress. We previously reported outcomes from the Acorn randomized trial to a common closing date (22.9 months of follow-up). This report summarizes results of extended followup to 5 years.

Methods: A total of 107 patients were enrolled in the no-mitral valve repair/replacement stratum including 57 in the CorCap treatment group and 50 in the control (optimal medical therapy alone) group. Patients were assessed every year, until completing 5 years of follow-up, for survival, adverse events, major cardiac procedures, New York Heart Association (NYHA) functional status, and echocardiograms, which were read at a core laboratory.

Results: Overall survivals were similar between the treatment and control groups, demonstrating no late adverse effect on mortality. The treatment group had significant reductions in left ventricular end-diastolic volume $(P=.029)$ as well as a small increase in sphericity index. More patients in the treatment group improved by at least 1 NYHA functional class $(P=.0005)$. There was no difference in rates of adverse events. In a subgroup of patients with an intermediate left ventricular end-diastolic dimension, there was a significant reduction in the Kaplan-Meier estimate of the freedom from the composite end point of death and major cardiac procedures $(P=.04)$.

Conclusions: These cumulative data demonstrate the sustained reverse remodeling of the left ventricle and the long-term safety and efficacy of the CorCap cardiac support device as an adjunctive therapy for patients with heart failure who remain symptomatic despite optimal medical therapy. (J Thorac Cardiovasc Surg 2012;143: 1036-42)

Earn CME credits at

http://cme.ctsnetjournals.org

Left ventricular (LV) remodeling is the pathophysiologic hallmark of heart failure (HF). It is characterized by ventricular dilation, a change from an ellipsoidal to a more spherical chamber shape, cardiomyocyte hypertrophy, interstitial fibrosis, and numerous alterations in biochemical and molecular functions. ${ }^{1} \mathrm{LV}$ remodeling is a strong predictor of survival in HF. All therapies with a positive impact on

From Acorn Cardiovascular, ${ }^{\mathrm{b}}$ St Paul, Minn; Henry Ford Hospital, ${ }^{\mathrm{c}}$ Detroit, Mich; Cleveland Clinic Foundation, ${ }^{\mathrm{d}}$ Cleveland, Ohio; University of Pennsylvania, ${ }^{\mathrm{e}}$ Phil-

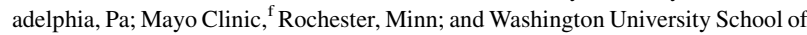
Medicine, ${ }^{\text {a }}$ St Louis, Mo.

Supported by a grant from Acorn Cardiovascular, Inc, St Paul, Minn.

Disclosures: Authors have nothing to disclose with regard to commercial support.

Received for publication Nov 20, 2010; revisions received May 6, 2011; accepted for publication June 7, 2011; available ahead of print July 15, 2011

Address for reprints: Douglas L. Mann, MD, Division of Cardiology, Washington University School of Medicine, 660 S. Euclid Ave, Campus PO Box 8086, St Louis, MO 63110-1093 (E-mail: dmann@dom.wustl.edu).

$0022-5223 / \$ 36.00$

Copyright $₫ 2012$ Published by Elsevier Inc. on behalf of The American Association for Thoracic Surgery

doi:10.1016/j.jtcvs.2011.06.014 clinical outcomes have a beneficial effect on remodeling, whereas therapies with a known deleterious effect on outcomes have adverse, neutral, or unknown effects on remodeling. ${ }^{2}$

Effective pharmacologic therapies in HF have operated either through a receptor-based mechanism (eg, angiotensinconverting enzyme inhibitors and beta-blockers) or through resynchronization (eg, biventricular pacemakers). To date, no therapy has specifically addressed the remodeling process or the important contributions of wall stress. The CorCap cardiac support device (CSD; Acorn Cardiovascular, Inc, St Paul, Minn) is a polyester mesh device that is fitted around the ventricles to provide circumferential diastolic support. In 3 different animal models of HF, implantation of the CSD resulted in favorable changes in LV size and function consistent with reverse remodeling. ${ }^{3-5} \mathrm{We}^{6}$ have previously reported the results of a randomized trial that enrolled 300 patients into 1 of 2 strata including the mitral valve repair/replacement (MVR) stratum, for patients undergoing MVR surgery, and the no-MVR stratum for patients who were solely provided optimal medical therapy. In that report, patients were followed up to a common closing date, which was a median of 22.9 months of follow-up. We now report results of follow-up extending to 5 years, including annual echocardiograms read by a central core laboratory. The 

Abbreviations and Acronyms
CSD = cardiac support device
$\mathrm{HF} \quad=$ heart failure
$\mathrm{LV} \quad=$ left ventricular
LVAD $=$ left ventricular assist device
MVR = mitral valve repair/replacement
NYHA $=$ New York Heart Association

inclusion of 2 different strata made it difficult to interpret the original report. Although both strata evaluated the impact of the CorCap device, the control group in the MVR strata also underwent mitral valve repair or replacement surgery, so that the incremental impact or benefit of the CorCap CSD was on top of the benefit associated with MVR surgery. In the noMVR stratum, the impact of the CSD was compared with medical therapy alone and therefore was the purer test of the CorCap hypothesis. This report will focus exclusively on the no-MVR stratum, in which patients were randomized to CorCap CSD implantation or optimal medical therapy alone.

\section{METHODS}

The Acorn Trial was a prospective and randomized study that enrolled 300 patients into 1 of 2 different strata. Patients with HF who had mitral regurgitation and a clinical indication for mitral valve surgery were enrolled into the MVR stratum $(\mathrm{n}=193)$. Results of this stratum and 5-year follow-up have been presented elsewhere and are not discussed in this report. ${ }^{7,8}$ Patients with HF who did not have significant mitral regurgitation $(\leq 2+)$ were enrolled into the no-MVR stratum. Patients were randomized to treatment (CorCap CSD plus optimal medical therapy) or control (optimal medical therapy alone).

\section{Patient Population}

Patients enrolled into the no-MVR stratum had New York Heart Association (NYHA) class III-IV HF of either ischemic or nonischemic etiology. Patients were between the ages of 18 and 80 years, with an LV ejection fraction of $35 \%$ or less, LV end-diastolic dimension of $60 \mathrm{~mm}$ or more (or an LV end-diastolic dimension indexed to body surface area $\geq 30 \mathrm{~mm} / \mathrm{m}^{2}$ ), 6-minute walk test distance of less than $450 \mathrm{~m}$, and acceptable laboratory and pulmonary function tests. All patients were on a stable and optimal medication regimen that included an angiotensin-converting enzyme inhibitor (or angiotensin receptor blocker if angiotensin-converting tension-intolerant) and beta-blockers (for at least 3 months) before randomization. Doses were titrated to recommended or highest tolerated levels and were kept stable for more than 1 month.

Exclusion criteria have been described previously. ${ }^{6}$ In particular, patients were excluded if they had any planned cardiac surgery, existing patent coronary artery bypass graft, a current or anticipated need for an LV assist device (LVAD), were on an active cardiac transplant list, had an anticipated need for heart transplantation within the next 2 years, had received biventricular pacing within the past 3 months, or had anticipated implantation of such a device within 12 months of enrollment. The protocol and informed consent form were reviewed and approved by the institutional review board before participation at each of the 29 clinical sites. Before baseline testing, all patients signed the informed consent form, which allowed follow-up through 5 years.

\section{Enrollment, Baseline Surgery, and Follow-up}

Baseline testing included chest radiograph, transthoracic echocardiogram, electrocardiogram, 6-minute walk test, maximal cardiopulmonary exercise test, quality of life tests, and assessment of NYHA class made by the site physician and by a blinded core laboratory.

After baseline testing, patients were randomized to either treatment or control. The control group did not undergo any surgery and were maintained on optimal medical therapy during follow-up. Crossover from control to treatment was not permitted. Patients in the treatment group underwent surgical implantation of the CorCap CSD using a standard sternotomy approach, as described previously. ${ }^{9}$

Follow-up was divided into 2 distinct phases. The pivotal efficacy phase started with the enrollment of the first patient and ended on a common closing date, which was defined as the date when the last enrolled patient had completed 12 months of follow-up. The last patient completed 12 months of follow-up on July 4, 2004, resulting in a median duration of follow-up of 22.9 months. During the pivotal efficacy phase, patients were seen for clinical assessment and repeat testing at 3 months, 6 months, and then every 6 months until the common closing date. The primary analysis of safety and efficacy of the CorCap device was based on data from the pivotal efficacy phase. ${ }^{6}$

After completion of the primary efficacy phase, patients entered an extended follow-up phase, in which long-term safety was monitored every year until 5 years after enrollment. Patient assessments during the extended follow-up phase included survival, adverse events, major cardiac procedures, and echocardiograms. Patients did not undergo follow-up echocardiograms after LVAD implantation or cardiac transplant. At each visit, patients were seen and evaluated by the principal investigator and research staff at each site.

All study echocardiograms were sent to the Echocardiography Core Laboratory at the Mayo Clinic. Each echocardiogram was evaluated by an extensive protocol specifically looking for evidence of constrictive physiology based on respiratory variations in mitral flow velocity, hepatic vein flow reversal, and superior vena cava forward flow velocity, based on the following definitions:

Definite constrictive physiology. Criteria 1, 2, and 3 are satisfied.

1. A $25 \%$ or greater change in mitral $E$ velocity with respiration, using the formula: $\%$ change $=(E \exp -\mathrm{E}$ insp $) / \mathrm{E}$ insp

2. A $40 \%$ or greater increase in diastolic hepatic vein flow reversal velocity with expiration compared with inspiratory diastolic flow reversal

3. Less than $20 \mathrm{~cm} / \mathrm{s}$ difference between systolic superior vena caval forward flow velocity with inspiration versus expiration.

Probable constrictive physiology. Criteria 2 and 3 are satisfied, or criterion 1 or 2 is satisfied with additional 2-dimensional features of constriction.

No constrictive physiology. There is no evidence of definite or possible constrictive physiology, as described above.

Further, in cases of adverse events such as hemodynamic compromise where constrictive physiology was considered a possibility, investigators were instructed to consider additional testing, including transesophageal echocardiography, computed tomography, magnetic resonance imaging, and cardiac catheterization with simultaneous right ventricular and LV pressure recordings.

Cumulative survival curves for the risk of death, adverse events, and major cardiac procedures were constructed according to the Kaplan-Meier method and significance assessed by the log- rank statistic. Comparisons of change from baseline for echocardiographic parameters were evaluated with longitudinal regression analysis, using a mixed-effects model in which follow-up visit was the repeated measure and the baseline value was the covariate.

Data were collected and stored in a database managed by Acorn Cardiovascular, Inc. All authors had full access to the data. 
TABLE 1. Baseline characteristics: no-MVR stratum

\begin{tabular}{|c|c|c|c|c|c|}
\hline & $\begin{array}{l}\text { All patients } \\
(\mathbf{n}=107)\end{array}$ & $\begin{array}{l}\text { Patients who completed } \\
\text { 5-year follow-up }(n=50)\end{array}$ & $\begin{array}{l}\text { Treatment } \\
(\mathbf{n}=29)\end{array}$ & $\begin{array}{l}\text { Control } \\
(\mathbf{n}=\mathbf{2 1})\end{array}$ & $P$ value (T vs C) \\
\hline Age (y) & 50.8 & 49.6 & 49.5 & 49.8 & NS \\
\hline \multicolumn{6}{|l|}{ Gender $(\%)$} \\
\hline Male & 72.9 & 70.0 & 65.5 & 76.2 & .05 \\
\hline Female & 27.1 & 30.0 & 34.5 & 23.8 & \\
\hline \multicolumn{6}{|l|}{ Race $(\%)$} \\
\hline White $(\%)$ & 73.8 & 78.0 & 79.3 & 76.2 & NS \\
\hline Black $(\%)$ & 20.6 & 18.0 & 17.2 & 19.1 & \\
\hline Other $(\%)$ & 5.6 & 4.0 & 3.5 & 4.8 & \\
\hline \multicolumn{6}{|l|}{ Etiology } \\
\hline Ischemic $(\%)$ & 16.8 & 16.0 & & & \\
\hline Idiopathic (\%) & 62.6 & 62.0 & & & \\
\hline Alcoholic $(\%)$ & 2.8 & 4.0 & & & \\
\hline Hypertensive (\%) & 9.4 & 12.0 & & & \\
\hline Viral $(\%)$ & 12.2 & 8.0 & & & \\
\hline Valvular (\%) & 0.9 & 2.0 & & & \\
\hline Other $(\%)$ & 7.5 & 4.0 & & & \\
\hline \multicolumn{6}{|l|}{ Medical treatment } \\
\hline ACE I or ARB (\%) & 96.3 & 98.0 & & & \\
\hline Beta-blocker (\%) & 94.4 & 96.0 & & & \\
\hline NYHA class III & 99.1 & 98.0 & 96.6 & 100 & NS \\
\hline LVEF (\%) & 26.1 & 27.0 & 25.3 & 29.5 & .053 \\
\hline LVEDD (mm) & 72.6 & 71.4 & 72.6 & 70.0 & NS \\
\hline LVEDV (mL) & 282.0 & 270.7 & 279.4 & 264.3 & NS \\
\hline 6MWT (m) & 334.7 & 338.9 & 322.8 & 361.1 & .065 \\
\hline MLHF (units) & 62.0 & 61.5 & 67.0 & 54.0 & .022 \\
\hline Peak $\mathrm{VO}_{2}\left(\mathrm{~mL} \cdot \mathrm{kg}^{-1} \cdot \mathrm{min}^{-1}\right)$ & 16.1 & 16.1 & 14.7 & 18.1 & .001 \\
\hline
\end{tabular}

$M V R$, Mitral valve repair/replacement; $T$, treatment; $C$, control; $N S$, not significant; $A C E$, angiotensin-converting enzyme; $A R B$, angiotensin receptor blocker; $N Y H A$, New York Heart Association; $L V E F$, left ventricular ejection fraction; $L V E D D$, left ventricular end-diastolic dimension; $L V E D V$, left ventricular end-diastolic volume; $6 M W T$, 6-minute walk test; $M L H F$, Minnesota Living with Heart Failure; $\mathrm{VO}_{2}$, Volume of oxygen utilization.

\section{RESULTS}

Table 1 summarizes the baseline characteristics of patients in the no-MVR stratum. The first column summarizes the 107 patients originally enrolled. ${ }^{6}$ The second column summarizes the baseline characteristics of the 50 patients who completed all 5 years of follow-up. In general, the patients completing 5 years of follow-up were similar to the patients who were originally enrolled with a mean age of 49.6 years. Most patients were male $(70.0 \%)$, had an idiopathic etiology $(62 \%)$, and were in NYHA class III $(98 \%)$. The mean ejection fraction was $27 \%$, the mean 6-minute walk distance was $338.9 \mathrm{~m}$, the mean Minnesota Living with Heart Failure score was 61.5 units, and the mean peak volume of oxygen utilization was $16.1 \mathrm{~mL} \cdot \mathrm{kg}^{-1} \cdot \mathrm{min}^{-1}$. Among the 50 patients who completed 5 years of follow-up, the treatment $(n=29)$ and control $(\mathrm{n}=21)$ groups were reasonably well matched. However, the control patients tended to be "less sick" on entry into the trial with a higher LV ejection fraction $(29.5 \%$ vs $25.3 \% ; P=.053)$, lower Minnesota Living with Heart Failure score (54.0 vs 67.0 units; $P=.022$ ), and higher peak volume of oxygen utilization (18.1 vs $\left.14.7 \mathrm{~mL} \cdot \mathrm{kg}^{-1} \cdot \min ^{-1} ; P=.001\right)$.
Of the original 107 patients enrolled in the no-MVR stratum, a total of 57 patients did not complete 5 years of follow-up owing to death $(\mathrm{n}=40)$, lost to follow-up $(\mathrm{n}=10)$, and withdrawn from study owing to patient/physician request $(n=7)$. The median duration of follow-up was 54.0 months, with 57.4 months in the treatment group and 51.3 months in the control group. The total follow-up was 384.9 patient-years.

Figure 1 summarizes 5 -year mortality rates. Over the 5 years of follow-up, there were 40 total deaths, including 19 deaths in the control group (overall mortality rate of $38 \%$ ) and 21 deaths in the treatment group (overall mortality rate of $36.8 \%$ ) The mortality curves between the treatment and control groups were very similar, demonstrating a lack of any late adverse effect on mortality. Causes of death were reported by the investigators to be primarily cardiovascular as described in Table 2 . There were no significant differences in the causes of death between the 2 groups. Neither this follow-up analysis nor the original Acorn Trial $^{6}$ was powered to detect a mortality benefit of the CorCap CSD.

Figure 2 summarizes the longitudinal regression analysis for the changes in LV end-diastolic volume. The control 


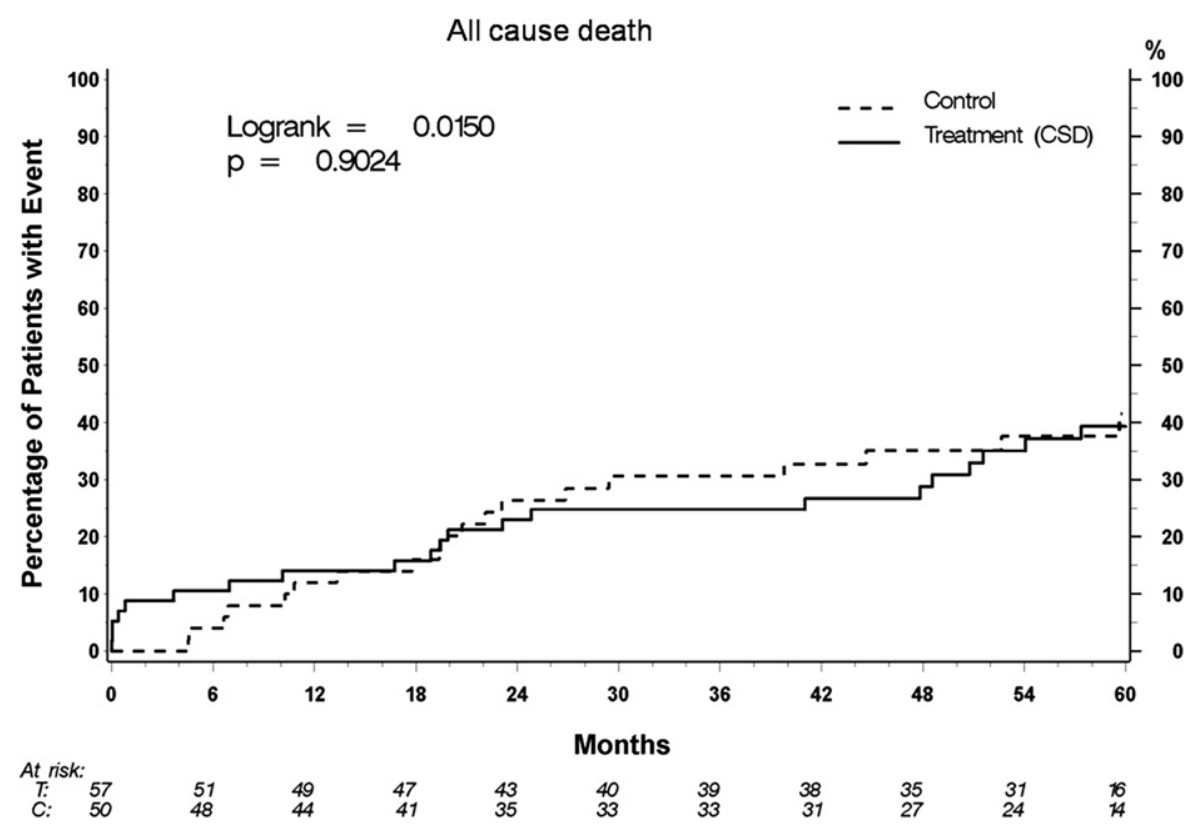

FIGURE 1. Kaplan-Meier mortality curve for the no-MVR stratum over 5 years of follow-up. Patients in the treatment group $(T)$ are in the solid line and patients in the control group $(C)$ are in the dotted line. The mortality curves between the treatment and control groups were similar, demonstrating the lack of any late adverse effect on mortality. $C S D$, Cardiac support device.

group did not demonstrate any consistent changes in LV size over 5 years of follow-up. In contrast, the CSD treatment group showed a progressive decrease in LV enddiastolic volume that was persistent over 5 years of follow-up. The differences between the treatment and control groups appeared to widen in the latter stages of followup. When averaged over 5 years, the treatment group LV end-diastolic volume was smaller by $28.9 \mathrm{~mL}$ compared with the control group $(P=.029)$. This represents an average decrease of $10.6 \%$ compared with the control group. For the treatment group, the average decrease in LV enddiastolic volume compared with baseline was $37.7 \%$. A similar pattern was observed for LV end-systolic volume (treatment group smaller than control group by $21.9 \mathrm{~mL}$; $P=.096)$. Since the changes in LV end-diastolic dimension and LV end-systolic dimension were similar, there were no significant differences in ejection fraction (estimated treatment difference 0.06 units; $P=$ not significant).

Figure 3 shows the changes in LV sphericity index (ratio of end-diastolic length/width). The control group had a small

TABLE 2. Causes of death

\begin{tabular}{lrcrrr}
\hline & \multicolumn{2}{c}{$\begin{array}{c}\text { Treatment group } \\
(\mathbf{n}=\mathbf{5 7})\end{array}$} & & \multicolumn{2}{c}{$\begin{array}{c}\text { Control group } \\
(\mathbf{n}=\mathbf{5 0})\end{array}$} \\
\cline { 2 - 3 } \cline { 6 - 7 } Causes of death & No. & Percent & & No. & Percent \\
\hline Cardiovascular & 14 & 66 & & 12 & 63.2 \\
Noncardiovascular & 3 & 14.3 & & 2 & 10.5 \\
Unknown & 1 & 4.7 & & 3 & 15.8 \\
Other/miscellaneous & 3 & 14.3 & & 2 & 10.5 \\
Total & 21 & & & 19 & \\
\hline
\end{tabular}

increase in sphericity index over 5 years of follow-up. The treatment group had a much larger increase in sphericity index and the difference compared with the control group appeared to increase over time, indicating return to a more physiologic ellipsoidal shape. The average difference between the treatment and control groups was 0.038 units, which was not statistically significant $(P=.26)$.

Table 3 summarizes the number of patients who had serious adverse events through 5 years of follow-up. There

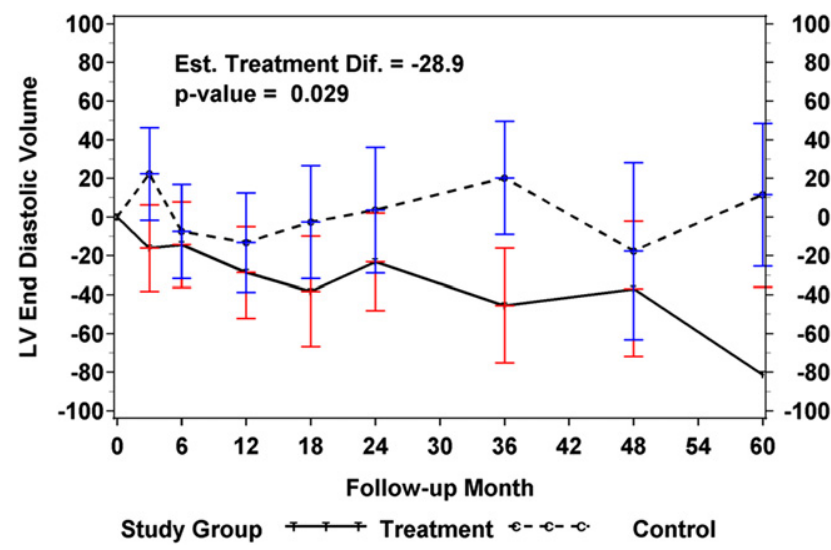

FIGURE 2. Longitudinal regression analysis for changes in left ventricu$\operatorname{lar}(L V)$ end-diastolic volume in the no-MVR stratum. The control group did not demonstrate any consistent changes in LV end-diastolic volume over 5 years. In contrast, the CSD treatment group showed a progressive decrease in LV end-diastolic volume that was persistent over 5 years. When averaged over 5 years, the LV end-diastolic volume of the treatment group was smaller by $28.9 \mathrm{~mL}$ than that of the control group $(P=.029)$. 


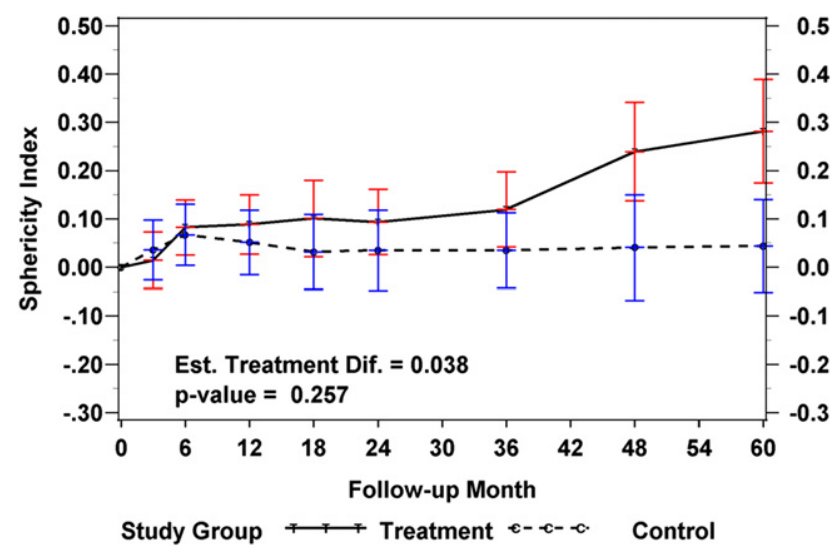

FIGURE 3. Longitudinal regression analysis for changes in left ventricular sphericity index in the no-MVR stratum. The treatment group had a much larger increase in sphericity index, indicating a change to a more ellipsoidal shape. The average 5-year treatment-control difference was 0.038 units, which was not statistically significant.

were $86 \%$ of patients with a serious adverse event in the control group, compared with $82.5 \%$ in the treatment group $(P=.79)$. The number of patients and the type of adverse events were not statistically different between the treatment and control groups.

The most commonly cited concern about CorCap CSD implantation is the late development of pericardial constriction. However, during the 5-year follow-up, no cases of pericardial constriction have been detected through adverse event reporting (hemodynamic compromise). Furthermore, we used annual surveillance echocardiograms that were read using a standardized evaluation protocol at the Mayo Core Laboratory. This protocol was very sensitive to detect possible changes in diastolic filling.

TABLE 3. Adverse events: no-MVR stratum-5-year follow-up

\begin{tabular}{lrrrrrr}
\hline & \multicolumn{2}{c}{$\begin{array}{c}\text { Treatment } \\
(\mathbf{n}=\mathbf{5 7})\end{array}$} & & \multicolumn{2}{c}{$\begin{array}{c}\text { Control } \\
(\mathbf{n}=\mathbf{5 0})\end{array}$} & \multicolumn{1}{r}{$\boldsymbol{P}$} \\
\cline { 2 - 3 } & No. & Percent & & No. & Percent & value \\
\hline Allergic response & 0 & 0.0 & & 0.0 & N/A \\
Arrhythmia & 19 & 33.3 & & 18 & 36.0 & .84 \\
Bleeding & 5 & 8.8 & & 8.0 & 1.00 \\
Hemodynamic Compromise & 39 & 68.4 & & 30 & 60.0 & .42 \\
Hepatic compromise & 0 & 0.0 & & 0 & 0.0 & N/A \\
Infection/pneumonia & 17 & 29.8 & & 13 & 26.0 & .67 \\
Myocardial infarction & 1 & 1.8 & & 1 & 2.0 & 1.00 \\
Neurologic deficit/stroke & 3 & 5.3 & & 5 & 10.0 & 0.47 \\
Peripheral thrombus/ & 2 & 3.5 & & 1 & 2.0 & 1.00 \\
$\quad$ embolism & & & & & \\
Pulmonary compromise & 11 & 19.3 & & 6 & 12.0 & .43 \\
Pulmonary embolism & 2 & 3.5 & & 1 & 2.0 & 1.00 \\
Renal compromise & 9 & 15.8 & & 3 & 6.0 & .13 \\
Other & 31 & 54.4 & & 23 & 46.0 & .44 \\
Any of the above SAEs & 47 & 82.5 & & 43 & 86.0 & .79 \\
\hline
\end{tabular}

$M V R$, Mitral valve repair/replacement; N/A, not available; SAEs, serious adverse events.

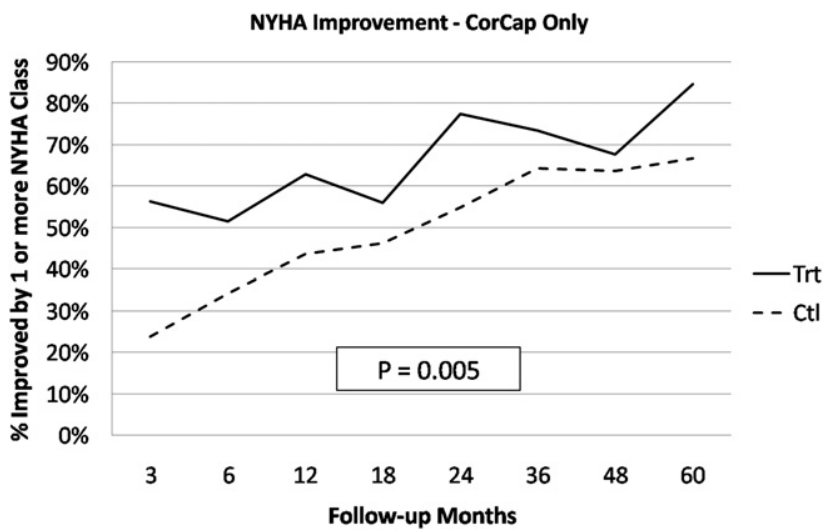

FIGURE 4. Longitudinal regression analysis for the percentage of patients with an improvement in New York Heart Association (NYHA) functional class of 1 class or more over 5 years of follow-up in the no-MVR stratum. At each time point, there were more patients in the treatment group who improved by at least 1 NYHA functional class. When averaged over 5 years, this difference was highly significant. Trt, Treatment; $C t$, control.

In the entire no-MVR cohort (107 patients), 20 patients at some point during follow-up (3-60 months) were deemed to have at least 1 "probable" or "definite" indication of constriction based on the echocardiographic measures. Of these 20 patients, 14 were in the treatment group and 6 were in the control group.

Thirteen patients had only 1 echo diagnosis of "probable" or "definite" constriction throughout the duration of followup (approximately $18 \%$ of completed visits), demonstrating that the majority of positive echo signals of constriction were isolated. In addition, these incidences were not supported by other clinical evidence, such as corresponding serious adverse event reports of hemodynamic compromise. Seven patients had multiple visits in which "probable" or "definite" evidence of constriction based on echo was noted. However, the evidence was not consistent through all follow-up visits and there were no corresponding clinical indications of hemodynamic compromise. Of these 7 patients, 4 were in the control group and 3 were in the treatment group.

NYHA functional class was assessed by site investigators at each of the follow-up visits. Figure 4 summarizes the percent of patients who improved by 1 NYHA functional class or more compared with their baseline status. Through 5 years of follow-up, $50 \%$ to $60 \%$ of patients in the control group improved by 1 functional class. This observation likely represents a "survivor" phenomenon, inasmuch as control patients with advanced HF who are able to survive 5 years are likely to be "responders." However, at each time point over 5 years of follow-up, there were more patients in the treatment group that improved by at least 1 NYHA functional class. When averaged over 5 years, this difference was highly significant $(P=.005)$.

Subgroup analysis was performed to determine whether etiology had any effect on the response to the CorCap 


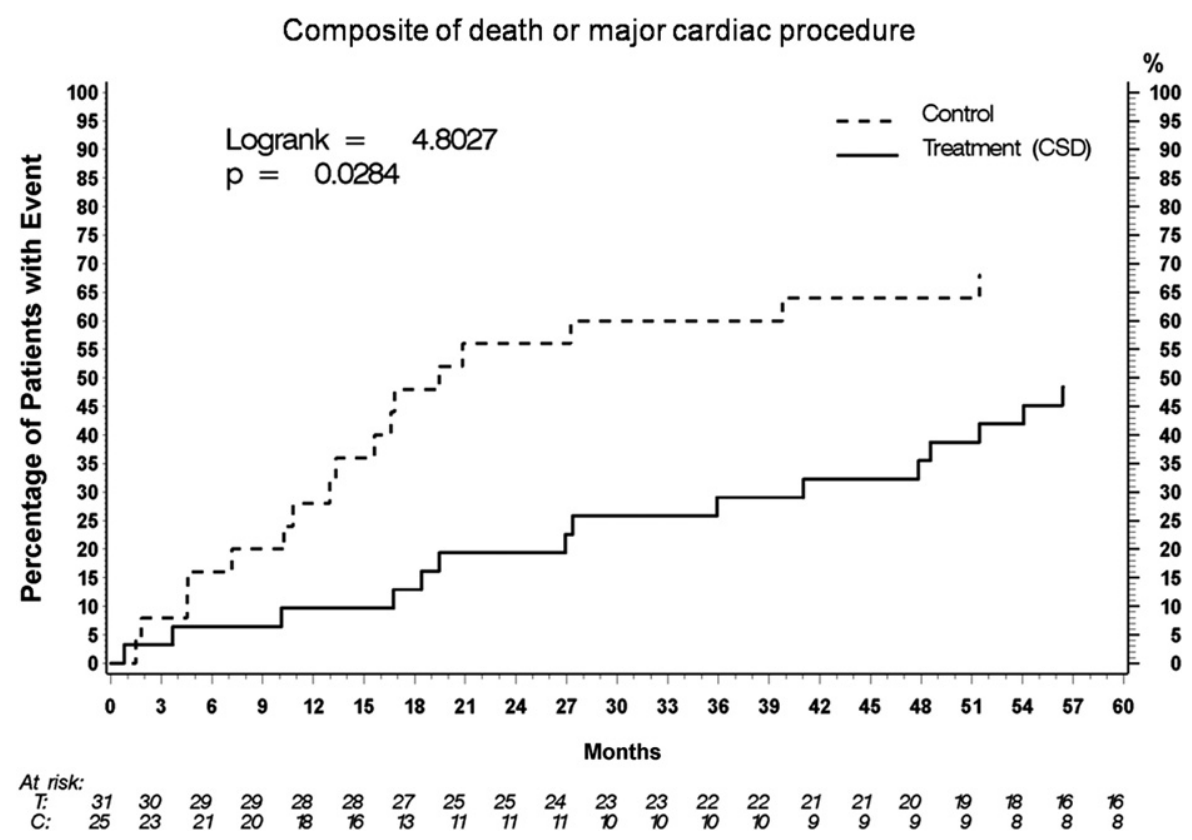

FIGURE 5. Kaplan-Meier estimate for the composite of death and major cardiac procedure for the focused cohort of the no-MVR stratum. There was a large separation between the groups that was maintained through 5 years of follow-up. The treatment group had fewer deaths, heart transplants, left ventricular assist devices, and biventricular pacemakers. CSD, Cardiac support device; $T$, treatment; $C$, control.

CSD, but there were no differences in the response in the patients with or without ischemic heart disease. However, only $16 \%$ of patients had ischemic heart disease, which may be too small to detect any differences in the response.

$\mathrm{We}^{6}$ previously reported on a post hoc analysis that identified a focused cohort as the group of patients who had the biggest treatment response (patients with an intermediate LV end-diastolic dimension indexed to body surface area that was $\geq 30 \mathrm{~mm} / \mathrm{m}^{2}$ and $\leq 40 \mathrm{~mm} / \mathrm{m}^{2}$ ). In the focused cohort, overall survival slightly favored the treatment group, but the difference was not statistically significant $(P=.73)$. Figure 5 shows the Kaplan-Meier estimate of the composite of death or major cardiac procedure for the focused cohort population $(n=56)$. There was a large separation between the groups that was maintained during the entire follow-up period $(P=.028)$. The entire patient population had a similar reduction in this composite end point, but the difference was not statistically significant $(P=.15)$. The treatment group had fewer heart transplants (4 vs 8 ), fewer LVADs ( 2 vs 4), and fewer biventricular pacemakers (12 vs 15) than did the control group. There had been concerns about the ability to perform these additional procedures in patients who had received the CorCap device. However, all the transplants, LVADs, and biventricular pacemakers were successfully implanted.

\section{DISCUSSION}

The principal findings from the 5-year follow-up of the no-MVR stratum is that the CorCap CSD is safe and associated with sustained beneficial effects on LV remodeling as well as sustained clinical benefits. The 5-year survival curves between the treatment and control groups were similar, confirming the absence of any late adverse effect on mortality. In addition, there was no difference in adverse events. Importantly, there were no signs of pericardial constriction, which is the most commonly cited concern of the CSD. Follow-up echocardiograms analyzed by a core laboratory over 5 years demonstrated persistence of benefit on LV remodeling with decreases in LV end-diastolic and end-systolic volumes and improvements in sphericity index. A greater percentage of patients improved by at least 1 NYHA functional class in the CSD treatment group. Finally, in a post hoc subgroup analysis of responders (focused cohort), there was a significant reduction in the combined end point of death and major cardiac procedures. Taken together, these 5-year follow-up data confirm the initial findings from the pivotal efficacy phase of the trial and suggest that the benefits persist over 5 years.

Previous reports of the Acorn Trial ${ }^{6,10}$ reported an analysis that combined both the MVR and the no-MVR strata. There were some difficulties in interpreting the data because the control group in the MVR stratum underwent mitral valve repair, whereas the control group in the no-MVR stratum were just maintained on optimal medical therapy. This report focuses exclusively on the no-MVR stratum, which was the purer test of the CorCap hypothesis since it compared the results of CorCap CSD versus medical therapy alone.

Collecting 5-year follow-up data on patients with HF is difficult. These patients are typically very ill, and many 
require additional cardiac procedures, may be enrolled in other clinical trials, or may die. However, these extended follow-up data are very important for the overall riskbenefit analysis of CorCap CSD implantation. The original report of the no-MVR stratum demonstrated a significant improvement in the primary composite end point $(P=.032)$, in which patients were considered improved, the same, or worsened on the basis of survival, the need for additional major cardiac procedures, and NYHA class. Advisory panels to the Food and Drug Administration reviewed all of the clinical trial data in June 2005 and December 2006. The panels supported the efficacy of the device but raised concerns about safety owing to the rate of perioperative mortality ( $4 / 51$ patients; $7.8 \%$ ). Further, there were concerns about the durability of the benefit and the potential appearance of constriction during later follow-up. The current data set is therefore a very important demonstration that the benefit is indeed durable and that there is not an increased incidence of late adverse events.

Data on the long-term effects of the CorCap CSD can be contrasted to the effects of cardiac resynchronization therapy. These specialized pacemakers are often associated with a dramatic and immediate improvement in symptoms and reverse remodeling of the left ventricle. However, reports have suggested that this initial benefit is not maintained in all patients long-term. ${ }^{11}$ In contrast, implantation of the CorCap CSD does not result in immediate clinical or echocardiographic benefit. However, the beneficial effects on LV size and shape are maintained for 5 years. To our knowledge, there are few reports of device therapy with documented beneficial effects on LV remodeling out of 5 years of follow-up. Another strength of the current study is that echocardiograms were read by a central core laboratory and the readers were blinded to treatment status.

A second-generation CSD was recently developed that can be implanted through a small thoracotomy incision. This alternate implant procedure should reduce many of the morbidities and complications associated with a full sternotomy. The feasibility and safety of this approach have been demonstrated in a study of 14 patients with the same baseline LV dimensions as the focused cohort (intermediate indexed LVend-diastolic diameter $>30$ and $<40 \mathrm{~mm} / \mathrm{m}^{2}$ ). A confirmatory trial on the safety and efficacy of the CorCap CSD using this thoracotomy delivery is pending.

This current study is limited by the small number of patients who completed 5 years of follow-up and by the fact that other important measures of patient functional class, such as quality of life and exercise tests, were not completed during the extended follow-up phase. However, patient functional class can be affected by noncardiac factors and comorbidities that may not be improved by the CSD. Further, all the clinical assessments were not blinded and are therefore influenced by physician and patient bias. Finally, these results could be influenced by surgeon reluctance to reoperate after CorCap CSD implantation.

In summary, data from 5-year follow-up of the no-MVR stratum of the Acorn Trial show that implantation of the CorCap CSD is safe with no apparent long-term complications or adverse effects on mortality. CSD therapy is associated with long-term sustained benefits on ventricular reverse remodeling, a sustained reduction in the combined end point of death/major cardiac procedures, and a sustained improvement in NYHA functional class. These cumulative data suggest that the CorCap CSD may represent a novel adjunctive therapy for stabilizing the progression of HF in patients who remain symptomatic despite optimal medical therapy.

\section{References}

1. Cohn JN. Structural basis for heart failure: ventricular remodeling and its pharmacological inhibition. Circulation. 1995;91:2504-7.

2. Mann DL, Bristow MR. Mechanisms and models in heart failure: the biomechanical model and beyond. Circulation. 2005;111:2837-49.

3. Sabbah HN, Sharov VG, Gupta RC, Mishra S, Rastogi S, Undrovinas AI, et al. Reversal of chronic molecular and cellular abnormalities due to heart failure by passive mechanical ventricular containment. Circ Res. 2003;93:1095-101.

4. Power JM, Raman J, Dornom A, Farish SJ, Burrell LM, Tonkin AM, et al. Passive ventricular constraint amends the course of heart failure: a study in an ovine model of dilated cardiomyopathy. Cardiovasc Res. 1999;44:549-55.

5. Pilla JJ, Blom AS, Brockman DJ, Bowen F, Yuan Q, Giammarco J, et al. Passive ventricular constraint improves myocardial left ventricular function and mechanics in a model of heart failure secondary to acute myocardial infarction. Circulation. 2002;106(12 suppl 1):I207-11.

6. Mann DL, Acker MA, Jessup M, Sabbah HN, Starling RC, Kubo SH. Clinical evaluation of the CorCap Cardiac Support Device in patients with dilated cardiomyopathy. Ann Thorac Surg. 2007;84:1226-35.

7. Acker MA, Bolling S, Shemin R, Kirklin J, Oh JK, Mann DL, et al. Mitral valve surgery in heart failure: insights from the Acorn Clinical Trial. J Thorac Cardiovasc Surg. 2006;132:568-77.

8. Acker MA, Jessup M, Bolling SF, Oh J, Starling RC, Mann DL, et al. Mitral valve repair in heart failure: five-year follow-up from the mitral valve replacement stratum of the Acorn randomized trial. J Thorac Cardiovasc Surg. 25 Jan 2011 [Epub ahead of print].

9. Oz MC, Konertz WF, Kleber FX, Mohr FW, Gummert JF, Ostermeyer J, et al. Global surgical experience with the Acorn cardiac support device. J Thorac Cardiovasc Surg. 2003;126:983-91.

10. Starling RC, Jessup M, Oh JK, Sabbah HN, Acker MA, Mann DL, et al. Sustained benefits of the CorCap Cardiac Support Device on left ventricular remodeling: three year follow-up results from the Acorn clinical trial. Ann Thorac Surg. 2007;84:1236-42.

11. St John Sutton MG, Plappert T, Hilpisch KE, Abraham WT, Hayes DL, Chinchoy E. Sustained left ventricular reverse structural remodeling with cardiac resynchronization at one year is a function of etiology. Circulation. 2006;113:266-77. 